\title{
Efficacy of Chelerythrine Against Mono- and Dual-Species Biofilms of Candida albicans and Staphylococcus aureus and Its Properties of Inducing Hypha-to-Yeast Transition of $C$. albicans
}

\author{
Weidong Qian ${ }^{1}{ }^{\mathbb{D}}$, Jianing Zhang ${ }^{1}$, Wenjing Wang ${ }^{1}$, Miao Liu ${ }^{1}$, Yuting Fu ${ }^{1}$, Xiang Li $^{1}$, \\ Ting Wang ${ }^{1, *(D)}$ and Yongdong $\mathrm{Li}^{2, *}$ \\ 1 School of Food and Biological Engineering, Shaanxi University of Science and Technology, \\ Xi'an 710021, China; qianweidong@sust.edu.cn (W.Q.); 1804070@sust.edu.cn (J.Z.); \\ 201504040311@sust.edu.cn (W.W.); 1804065@sust.edu.cn (M.L.); 201504040401@sust.edu.cn (Y.F.); \\ lixiang@sust.edu.cn (X.L.) \\ 2 Ningbo Municipal Center for Disease Control and Prevention, Ningbo 315010, China \\ * Correspondence: wangtingsp@sust.edu.cn (T.W.); liyd@nbcdc.org.cn (Y.L.); Tel.: +86-29-86168583 (T.W.)
}

Received: 6 February 2020; Accepted: 1 April 2020; Published: 2 April 2020

\begin{abstract}
Candida albicans and Staphylococcus aureus specifically often resulted in biofilm-associated diseases, ranging from superficial mucosal to life-threatening systemic infections. Recent studies reported that chelerythrine displayed antimicrobial activities against a few microorganisms, but its effects on mono- and dual-species biofilms of C. albicans and S. aureus have never been reported. The purpose of this study was to evaluate the efficacy of chelerythrine against mono- and dual-species biofilms, and explore its effect on the hyphal growth and the hypha-to-yeast transition of C. albicans. The results showed that minimum inhibitory concentrations (MICs) and minimum biofilm inhibitory concentration ( $\mathrm{MBIC}_{90 \mathrm{~S}}$ ) of chelerythrine against planktonic cells of mono-species were 4 and $2 \mu \mathrm{g} / \mathrm{mL}$, while the MIC and MBIC $_{90}$ were 6 and $3 \mu \mathrm{g} / \mathrm{mL}$ for dual-species. Meanwhile, the decrease in three matrix component levels and tolerance to antibiotics of biofilms formed by mono- and dual-species exposed to chelerythrine were confirmed by a confocal laser scanning microscope, in conjugation with five fluorescent dyes and a gatifloxacin diffusion assay. Moreover, C. albicans and S. aureus mono-species showed a 96.4, and 92.3\% reduction, respectively, in 24-h preformed biofilm biomass in the presence of $128 \mu \mathrm{g} / \mathrm{mL}$ of chelerythrine. Similarly, preformed $(24 \mathrm{~h})$ dual-species biofilm biomass also displayed a significant reduction $(90.7 \%)$ when treated with $192 \mu \mathrm{g} / \mathrm{mL}$ chelerythrine. Chelerythrine inhibited hyphae formation of C. albicans at $4 \mu \mathrm{g} / \mathrm{mL}$, and C. albicans in hypha-form can be converted into yeast-form at $8 \mu \mathrm{g} / \mathrm{mL}$ of chelerythrine. Therefore, chelerythrine shows promise as a potential antimicrobial and antibiofilm agent for clinical effective treatments of mono- and mixed-species and/or biofilm-associated infections.
\end{abstract}

Keywords: Candida albicans; Staphylococcus aureus; chelerythrine; dual-species biofilm; antibiofilm activity

\section{Introduction}

Candida accounts for $70 \%$ to $90 \%$ of all human fungal infections and can be associated with devastating consequences, particularly in intensive care units where mortality rates reach $40 \%$ [1,2]. Oral candidiasis is one of the observed mostly human opportunistic fungal infections of the oral cavity, caused by an overgrowth of Candida species, the most common being Candida albicans [3]. C. albicans, a dimorphic fungal organism, is present primarily in the oral cavity in a non-pathogenic yeast state in 
about one half of healthy individuals, but, under nutrition-rich conditions, has the ability to switch to a disease-causing hyphae form [4]. Under poor oral health conditions, pathogens including methicillin resistant Staphylococcus aureus and Pseudomonas aeruginosa were easy to colonize in the oral cavity [5]. Oral microorganisms adhere to a surface and evolve to persist in the varying environments in protective biofilms [6]. Notably, in a diverse range of pathological niches, C. albicans often coexist with S. aureus, which are often co-isolated in cases of biofilm-associated infections, and have been implicated in blood, urinary tract, and burn wound infections, as well as infections of medical devices such as central venous catheters [7]. Specifically, C. albicans partners with S. aureus to enhance bacterial colonization and biofilm formation, where fungi can provide a scaffold for the biofilm formation of bacteria, thus allowing bacteria to survive in hostile environments or antibiotic treatments, and exacerbating mucosal tissue infection and destruction [8,9]. The complexity of polymicrobial biofilm-mediated infections presents an additional challenge to explore effective treatment strategies.

Biofilms are self-adhering communities of microorganisms embedded in a matrix of extracellular polymeric substances attached to an interface [10]. Biofilm formation involves several steps, namely the attachment to biotic or abiotic surfaces, maturation, and dispersal of mature biofilm and has long been recognized serve as a reservoir for pathogenic cells. Thus, biofilms can cause septicemia, and evolve into invasive systemic infections of organs and tissues [11]. The multifactorial nature of biofilms caused by mixed fungal-bacterial infections, along with the rapid emergence of antimicrobial resistance in pathogenic fungi and bacteria, imposes great challenges for the use of conventional antimicrobials, and further limits therapeutic options [12]. To prevent and address such stubborn infections caused by dual-species biofilm, effective anti-biofilm development and mature biofilm eradication agents are urgently needed. Nowadays, promising antimicrobials derived from natural extracts and isolated compounds from plants have attracted a lot of attention, due to their potential therapeutic value in various diseases and as a source of novel drugs.

Chelerythrine, a benzo-c-phenanthridine alkaloid extracted from Bocconia cordata, has been utilized as a traditional medicine. Previous studies reported that chelerythrine exhibits various biological and pharmacological properties, including anti-cancer, anti-inflammatory, insecticide, anti-fibrosis activities, etc. $[13,14]$. A few recent studies reported antimicrobial activities of chelerythrine against $C$. albicans, S. aureus, Escherichia coli, and Aeromonas hydrophila [15-18]. However, the inhibitory and scavenging effects of chelerythrine against mono- and dual-species biofilms of $C$. albicans and $S$. aureus, as well as its effect on the hyphal growth and mature hypha switch of $C$. albicans, have yet to be fully investigated. Thus, the main purpose of this study was to evaluate the antibiofilm and mature biofilm eradication activities of chelerythrine against mono- and dual-species biofilms of $C$. albicans and S. aureus.

\section{Materials and Methods}

\subsection{Reagents}

Chelerythrine of HPLC grade was supplied by Chengdu Pulis biological science and Technology Co., Ltd. (Chengdu, China). Chelerythrine was dissolved in $1 \%$ dimethyl sulfoxide (DMSO, Sigma-Aldrich, St. Louis, MI, USA) and distilled water prior to use. SYTO 9, Film Tracer SYPRO Ruby, wheat germ agglutinin (WGA), propidium iodide (PI), and lipophilic membrane FM 4-64 dyes were purchased from Invitrogen (Thermo Fisher Scientific, Waltham, MA, USA). All other chemicals and solvents used were of analytical grade.

\subsection{Bacterial Strains and Cultural Conditions}

Candida albicans SC5314 and S. aureus ATCC25923 strains were employed and routinely grown in yeast peptone dextrose (YPD; $10 \mathrm{~g} / \mathrm{L}$ of yeast extract, $20 \mathrm{~g} / \mathrm{L}$ of peptone, and $20 \mathrm{~g} / \mathrm{L}$ of glucose; Sigma-Aldrich, USA) and tryptic soy broth (TSB; Sigma-Aldrich, St. Louis, USA) medium under aerobic conditions at $37^{\circ} \mathrm{C}$ for $18-24 \mathrm{~h}$ in a shaking incubator $(180 \mathrm{rpm})$, respectively. The fungal and bacterial stocks were stored at $-80^{\circ} \mathrm{C}$ and supplemented with $25 \%$ glycerol (Sigma-Aldrich, St. Louis, 
USA) as a cryoprotectant. For mixed biofilm formation, C. albicans SC5314 and S. aureus ATCC25923 inoculum in saline solution at approximately $1 \times 10^{6} \mathrm{CFU} / \mathrm{mL}$ were cultured on $1 \mathrm{~cm} \times 1 \mathrm{~cm}$ glass coverslips in individual wells of a 24 -well plate at $37^{\circ} \mathrm{C}$ for $24 \mathrm{~h}$ in a shaking incubator (100 rpm).

\subsection{Minimal Inhibitory Concentrations (MICs)}

To determine the MICs of chelerythrine against C. albicans SC5314 and S. aureus ATCC25923, broth microdilution was performed according to the Clinical and Laboratory Standards Institute (CLSI) guidelines M27-A3 [19] and M7-M9 [20], respectively, with minor modifications. Briefly, overnight fungal and bacterial cultures were suspended in YPD and TSB at a final density of approximately $1 \times 10^{6}$ colony forming unit $(\mathrm{CFU}) / \mathrm{mL}$, transferred into individual wells of the 96-well microtiter plate (Nunc, Copenhagen, Denmark), and then incubated with final concentrations of chelerythrine, ranging from 2 to $512 \mu \mathrm{g} / \mathrm{mL}$. Fluconazole $(64 \mu \mathrm{g} / \mathrm{mL})$ and vancomycin $(16 \mu \mathrm{g} / \mathrm{mL})$ were employed as the positive control, while $1 \%$ DMSO was used as negative control group. After incubation at $37^{\circ} \mathrm{C}$ for $24 \mathrm{~h}$ without shaking, the growth of C. albicans SC5314 and S. aureus ATCC25923 was examined by measuring the absorbance at $600 \mathrm{~nm}\left(\mathrm{OD}_{600}\right)$ using a Multiskan $\mathrm{GO}$ plate reader (Thermo Fisher Scientific, Waltham, MA, USA). The MIC was determined from independent triplicate assays and defined as the lowest concentration, at which microbial cell growth was not detected.

\subsection{Minimum Biofilm Inhibitory Concentrations (MBIC $90 \mathrm{~s}$ )}

The minimum biofilm inhibitory concentration (MBIC ${ }_{905}$ ), which is defined as the lowest concentration of antimicrobials that results in the inhibition of $90 \%$ biofilm formation, was quantified by the crystal violet $(\mathrm{CV})$ assay and CFU enumeration. For the $\mathrm{CV}$ assay, overnight cultures of mono- and dual-species grown in YPD were firstly diluted to an inoculum dose of approximately $1 \times 10^{6} \mathrm{CFU} / \mathrm{mL}$. Then the dilution of C. albicans SC5314 and S. aureus ATCC25923 mono- and dual-species with a ratio of 1:1 was incubated in individual wells of a 96-well microtiter plate and treated with chelerythrine at $0,1 / 16,1 / 8,1 / 4,1 / 2$, and MIC for $24 \mathrm{~h}$. After incubation, the biofilms formed in individual wells were fixed with $200 \mu \mathrm{L}$ of methanol after being gently washed three times with $5 \mathrm{~mL}$ phosphate buffered saline (PBS; $2.7 \mathrm{mM} \mathrm{KCl}, 1.5 \mathrm{mM} \mathrm{KH}_{2} \mathrm{PO}_{4}, 136.9 \mathrm{mM} \mathrm{NaCl}, 8.9 \mathrm{mM} \mathrm{Na}_{2} \mathrm{HPO}_{4}, \mathrm{pH}$ 7.4). Then, the samples were stained with $200 \mu \mathrm{L}$ of $0.1 \%$ crystal violet solution for $15 \mathrm{~min}$. Followed by a $15 \mathrm{~min}$ inoculation, each well was washed with distilled water and added with $200 \mu \mathrm{L}$ of acetic acid (33\%, $v / v)$, to dissociate the crystal violet attached to bacterial cell walls. For each sample, the absorbance at $570 \mathrm{~nm}$ was recorded by a microplate reader. Moreover, to calculate the number of viable fungal or bacterial cells, followed by treatment, the washed cells were then removed from each glass coverslip by washing with $1 \mathrm{~mL}$ of $10 \mathrm{mM}$ PBS, then transferred into prelabeled microfuge tubes and sonicated for $10 \mathrm{~min}$. The resulting cell suspensions of dual-species biofilms were diluted ten-fold and cultured on a sabouraud dextrose agar medium (SDA; Sigma-Aldrich, St. Louis, USA), supplemented with chloramphenicol (50 mg/L) (selective for C. albicans) and mannitol salt agar (MSA; Sigma-Aldrich, St. Louis, USA) (selective for S. aureus). Similarly, ten-fold dilutions of $C$. albicans and S. aureus biofilms were plated on SDA and MSA, respectively. After an additional $24 \mathrm{~h}$ incubation at $37^{\circ} \mathrm{C}$, the fungal and bacterial colonies were counted.

\subsection{Biofilm Inhibition Assay}

The inhibitory effects of chelerythrine on the biofilm formation were observed by field emission scanning electron microscopy (FESEM; Nova Nano SEM-450, FEI, Hillsboro, OR, USA) and confocal laser scanning microscopy (CLSM; Zeiss LSM 880 with Airyscan), as described previously [21]. Approximately $1 \times 10^{6} \mathrm{CFU} / \mathrm{mL}$ of mono-species and dual-species were cultured on the glass coverslips in individual wells of a 24-well microtiter plate, and treated with chelerythrine at $0,1 / 8,1 / 4$, and 1/2 MIC at $37^{\circ} \mathrm{C}$ for $24 \mathrm{~h}$. For FESEM analysis, followed by treatment, the glass coverslips with biofilms were kept in sterile water containing 2.5\% glutaraldehyde ( $v / v$; Sigma-Aldrich, USA) at $-4{ }^{\circ} \mathrm{C}$ for $2 \mathrm{~h}$. Then, biofilms were rinsed three times with $10 \mathrm{mM}$ PBS (pH 7.0) and dehydrated in water-alcohol solutions at 
various alcohol concentrations of 30\%, 50\%,70\%, 90\%, and 100\% for 10 min each. Finally, the resulting samples were characterized using a FESEM. Similarly, for CLSM analysis, the glass coverslips with biofilms were washed three times with $0.9 \%$ sodium chloride $(\mathrm{NaCl})$ and incubated with $2.5 \mu \mathrm{MSYTO} 9$. After incubation at $25^{\circ} \mathrm{C}$ for $15 \mathrm{~min}$, the biofilms were examined by CLSM, where the fluorescence were measured at excitation/emission wavelengths of $485 / 542 \mathrm{~nm}$ for SYTO 9.

\subsection{Biofilm Composition by CLSM}

The composition changes of 24-h biofilms of mono- or dual-species were observed by CLSM. Briefly, approximately $1 \times 10^{6} \mathrm{CFU} / \mathrm{mL}$ of mono-species and dual-species were cultured on the glass coverslip in each well of a 24-well microtiter plate and treated with chelerythrine of different concentrations $(0,1 / 8,1 / 4$, and $1 / 2 \mathrm{MIC})$ for $24 \mathrm{~h}$ at $37^{\circ} \mathrm{C}$. Then, the resulting samples were exposed to the following five types of dyes: (I) SYTO 9 dye, which stains nucleic acids; (II) Film Tracer SYPRO Ruby Biofilm Matrix stain, which labels most classes of proteins [22]; (III) WGA conjugated with Oregon Green, which stains $\mathrm{N}$-acetyl-D-glucosamine residues [23]; (IV) PI dye that stains nucleic acids; (V) FM 4-64 dye, which stains lipophilic membrane. The fluorescence of dyes was measured using the following combination of excitation and emission wavelengths: $476 \mathrm{~nm} / 500-520 \mathrm{~nm}$ for SYTO 9, $405 \mathrm{~nm} / 655-755 \mathrm{~nm}$ for SYPRO Ruby, $459 \mathrm{~nm} / 505-540 \mathrm{~nm}$ for WGA, $535 \mathrm{~nm}$ and $617-635 \mathrm{~nm}$ for PI, and for and 479nm/565-588 nm for FM 4-64, respectively. After each staining step, the biofilms were gently rinsed with $10 \mathrm{mM}$ PBS. The biofilm images were acquired using a CLSM. Red/green fluorescence ratios to assess extracellular matrix components, including eDNA, proteins, and polysaccharides were measured on PI/SYTO 9, SYPRO Ruby/SYTO 9, and FM 4-64/WGA images, respectively, with KS 400 version 3.0 software (Carl Zeiss, Inc., Jena, Germany).

\subsection{Antibiotics Diffusion within Biofilms}

To assess the diffusion capability of antibiotics in biofilms formed in the presence of chelerythrine, gatifoxacin diffusion within biofilms was measured based on its intrinsic fluorescence by CLSM [24]. Biofilms of mono- and dual-species were prepared in the same manner as described above on the glass coverslips placed inside the 24-well plate (Corning, NY, USA). The resulting biofilms were gently washed three times with $10 \mathrm{mM}$ PBS, and a final concentration of $80 \mu \mathrm{g} / \mathrm{mL}$ gatifoxacin was added and further incubated for $5 \mathrm{~h}$ at $37^{\circ} \mathrm{C}$. Then, a final concentration of $3 \mu \mathrm{M}$ SYTO 9 was added to visualize the gatifoxacin diffusion within biofilms. Followed by $15 \mathrm{~min}$ incubation, the samples were rinsed three times with $10 \mathrm{mM}$ PBS to remove nonpenetrated gatifoxacin and observed using a CLSM. For an in-depth understanding of gatifloxacin diffusion scope and depth in biofilms, representative images in the $\mathrm{z}$ axis were obtained. At least three random fields were visualized for each biofilm.

\subsection{Minimal Biofilm Eradication Concentration}

To investigate the potential ability of chelerythrine to eradicate the preformed biofilms of monoand dual-species, the preformed biofilm eradication assay was carried out in a 24-well microtiter plate, as previously described [25]. $24 \mathrm{~h}$ biofilms of mono-species and dual-species preformed on the glass coverslips in each well were treated with chelerythrine at different concentrations $(0,8,16$, and $32 \mathrm{MIC})$ for $5 \mathrm{~h}$ at $37^{\circ} \mathrm{C}$. Afterward, the biofilm was observed using a FESEM. Meanwhile, the biofilm biomass was measured by the crystal violet staining assay. Further, CFU recovery for viability was performed in treated biofilms as described above.

\subsection{Effect of Chelerythrine on C. albicans Hyphal Growth and Mature Hyphae}

Hyphal growth inhibition was examined according to hyphal length analysis. Candida albicans SC5314 was grown in YPD broth at $37^{\circ} \mathrm{C}$ for $16 \mathrm{~h}$ in a shaking incubator at $180 \mathrm{rpm}$, washed twice with $10 \mathrm{mM}$ PBS, and diluted to a concentration of approximately $1 \times 10^{6} \mathrm{CFU} / \mathrm{mL}$, using serum-free Roswell Park Memorial Institute 1640 medium (RPMI 1640; Invitrogen, Grand Island, NY, USA). For hyphal growth analysis, $100 \mu \mathrm{L}$ of $C$. albicans cell dilution (approximately $1 \times 10^{8} \mathrm{CFU} / \mathrm{mL}$ ), $700 \mu \mathrm{L}$ of fresh 
RPMI 1640 medium, and $200 \mu \mathrm{L}$ of different concentrations of chelerythrine $(0,1 / 4,1 / 2$, and MIC) were mixed in each well of a 24-well culture plate, and cultured at $37{ }^{\circ} \mathrm{C}$ in $5 \% \mathrm{CO}_{2}$ for $5 \mathrm{~h}$. Thereafter, hyphae of C. albicans SC5314 were visualized using a FESEM. For mature hyphae analysis, $100 \mu \mathrm{L}$ of C. albicans SC5314 cell dilution and $900 \mu \mathrm{L}$ of fresh RPMI 1640 medium were mixed in each well of a 24-well culture plate, and cultured at $37^{\circ} \mathrm{C}$ in $5 \% \mathrm{CO}_{2}$ for $36 \mathrm{~h}$. Subsequently, the resulting samples were treated with different concentrations of chelerythrine $(0,1 / 2,1$, and $2 \mathrm{MIC})$ for $4 \mathrm{~h}$ at $37^{\circ} \mathrm{C}$. Subsequently, the samples were observed by a FESEM.

\subsection{Statistical Analysis}

All experiments were performed in triplicate. The data were expressed as mean values with the corresponding standard deviations (SD). Statistical analyses were performed with the statistical program SPSS software (SPSS 8.0 for Windows). Analysis of variance (ANOVA) was carried out to examine any significant differences $(p \leq 0.01)$.

\section{Results}

3.1. Minimum Inhibitory Concentrations $\left(\mathrm{MIC}_{S}\right)$ and Minimum Biofilm Inhibitory Concentration (MBIC $\left.90 \mathrm{~S}\right)$ of Chelerythrine against $C$. albicans and S. aureus

The MICs and $\mathrm{MBIC}_{905}$ of chelerythrine against C. albicans, S. aureus mono- and dual-cultures were presented in Table 1. Chelerythrine displayed potent antibacterial activities to mono- and dual-cultures. The MICs and $\mathrm{MBIC}_{905}$ of chelerythrine against $C$. albicans and S. aureus single cultures were 4 and $2 \mu \mathrm{g} / \mathrm{mL}$. In addition, the MICs and $\mathrm{MBIC}_{905}$ of chelerythrine against the mixed culture of C. albicans and $S$. aureus were 6 and $3 \mu \mathrm{g} / \mathrm{mL}$.

Table 1. Minimum Inhibitory Concentrations (MIC)s and Minimum Biofilm Inhibitory Concentration $\left(\mathrm{MBIC}_{90 \mathrm{~S}}\right)$ of chelerythrine against mono- and dual-species planktonic cells of Candida albicans and Staphylococcus aureus.

\begin{tabular}{ccc}
\hline Strain & MIC $_{\mathbf{S}}(\mu \mathrm{g} / \mathrm{mL})$ & MBIC $_{\mathbf{9 0 5}}(\mu \mathrm{g} / \mathrm{mL})$ \\
\hline Candida albicans SC5314 & 4 & 2 \\
Staphylococcus aureus ATCC25923 & 4 & 2 \\
Dual species & 6 & 3 \\
\hline
\end{tabular}

\subsection{Chelerythrine Decreased the Biofilm Formation of C. albicans and S. aureus Mono- and Dual-Species}

The biofilm biomass of mono- and dual-species exposed to chelerythrine was measured using the crystal violet cell viability assay. As demonstrated in Figure 1A, the addition of chelerythrine reduced remarkably the biofilm biomass of the mono- and dual-species in a concentration-dependent manner. When $C$. albicans exposed to chelerythrine at the $1 / 8 \mathrm{MIC}$, there is a significant difference in the biofilm biomass between the treated and untreated group $(p<0.01)$. By contrast, the biofilm biomass of $S$. aureus mono- and dual-species in 1/4 MIC chelerythrine-treated groups displayed a significant difference, compared with the untreated group. The biofilm biomass of mono- and dual-species was further downregulated significantly $(p<0.001)$ after exposure to chelerythrine at $1 / 2$ MIC or MIC, compared to the untreated control. The results showed that chelerythrine had an excellent inhibitory effect on the biofilm formation of C. albicans and S. aureus mono- and dual-species, and $\mathrm{MBIC}_{90}$ of chelerythrine against $C$. albicans and $S$. aureus mono-species biofilms were $2 \mu \mathrm{g} / \mathrm{mL}$, while the $\mathrm{MBIC}_{90}$ of chelerythrine against dual-species biofilms was $3 \mu \mathrm{g} / \mathrm{mL}$ (Table 1 ). Moreover, after the biofilm formation of dual species for $24 \mathrm{~h}$, the cell number of control samples was $9.2 \log _{10} \mathrm{CFU} /$ coverslip for S. aureus and $7.2 \log _{10} \mathrm{CFU} /$ coverslips for C. albicans, whereas overall a 6.5 to $4.1 \log _{10} \mathrm{CFU} / \mathrm{coverslips}$ reduction was obtained for S. aureus and C. albicans, respectively, when treated with 1/4 MIC chelerythrine (Figure 1B). A similar trend was observed with a decline from 7.8 to $4.5 \log _{10}$ CFU/coverslips for $1 / 4$ MIC chelerythrine-treated S. aureus and C. albicans dual-species biofilm 
cells. For $C$. albicans and S. aureus mono- and dual-species biofilms tested, at 1/2 MIC chelerythrine concentrations, only $3.1 \log _{10} \mathrm{CFU} /$ coverslips or even less was observed. After treatment with MIC concentration, no survivors of either microbial cell were detected (Figure 1B).
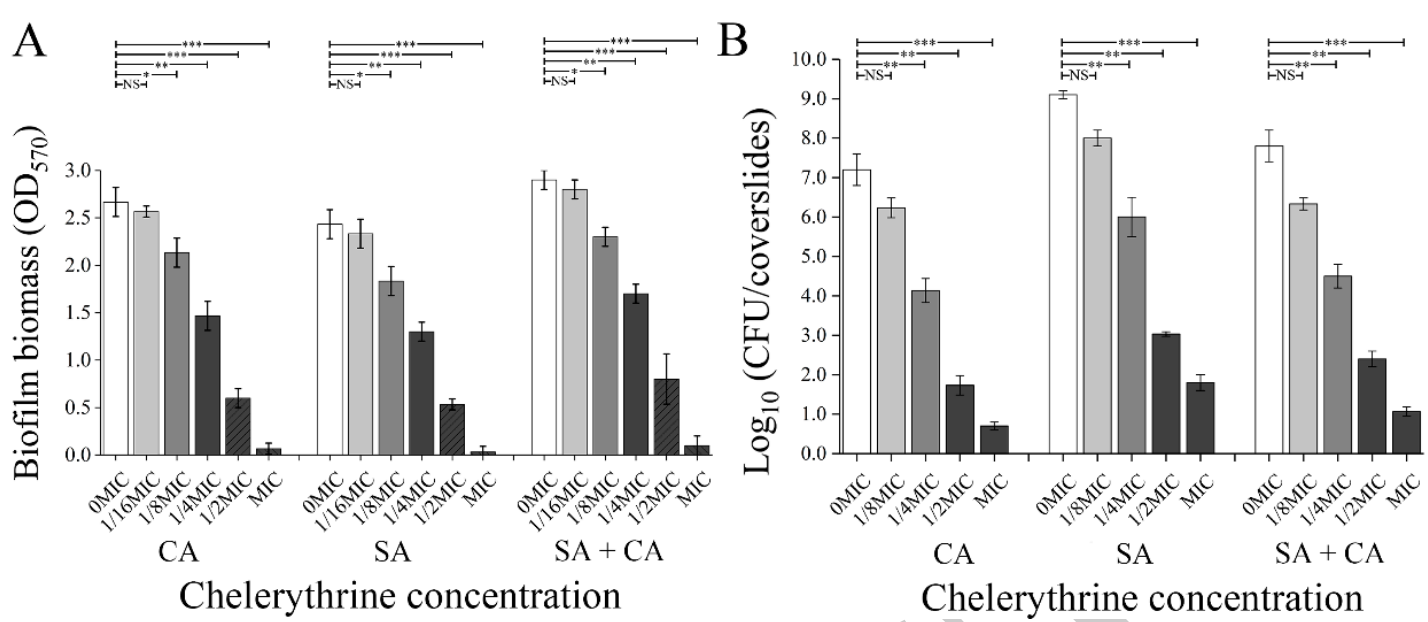

Figure 1. Assessment of different concentrations of chelerythrine on C. albicans (CA) and S. aureus (SA) mono- and dual-species (SA+CA) biofilm biomass by crystal violet staining and cell enumeration by colony count. (A) The biofilm biomass was determined using the crystal violet staining assay. Values represent the means of triplicate measurements. (B) Biofilm production analysis was performed by cell enumeration $\left(\log _{10} \mathrm{CFU} /\right.$ coverslips). Bars represent the standard deviation $(n=3) .{ }^{*} p<0.05$; ${ }^{* *} p<0.01 ;{ }^{* * *} p<0.001 ; \mathrm{NS}$, not significant.

3.3. Sub-MIC Chelerythrine Changed the Biofilm Community Structure of C. albicans and S. aureus Mono- and Dual-Species

The basic biofilm structure of mono- and dual-species exposed to sub-MIC chelerythrine was analyzed by FESEM and CLSM. As demonstrated in Figure 2A, mono- and dual-species cells formed strong and sticky aggregates in the absence of chelerythrine. Especially, Figure 2A revealed also S. aureus to be distributed along the round C. albicans cells throughout the entire biofilm architecture, indicating the potentially synergistic relationship during polymicrobial growth. In contrast, subjecting mono- and dual-species cells to chelerythrine sub-MIC resulted in a less dense and loose biofilm network. Obviously, in the case of $1 / 2$ MIC chelerythrine-treated group, the $C$. albicans mono- and dual-species biofilm structures were missing, and only individual cells can be viewed with disruption of the complex structure of the biofilms, but for S. aureus strains, distinct grape-like clusters appeared with less cell aggregations compared to the untreated group. In addition, in the treated group with chelerythrine at $1 / 8$ MIC, biofilms of pure- and mixed-culture were generated by a dense and tight network of bacterial cells, except the treated groups had relatively low biofilm volumes.

We further examined the architecture of the biofilms in the presence or absence of chelerythrine by staining with SYTO 9 dyes to detect live cells using a CLSM. As shown in Figure 2B, a dense cluster composed largely of macrocolonies was present in the untreated C. albicans pure-culture group, while the biofilms cultivated in presence of chelerythrine appeared looser and more dispersed with the rise of the concentration used, suggesting that existing cell-cell adhesiveness inside the biofilm decreased over concentration. Similarly, in the absence of chelerythrine, a luxuriant homotypic biofilm was formed by $S$. aureus pure culture, whereas the less dense biofilm with the bubble-like structure was observed in the 1/2 MIC treated group, and less coverage gave way to loss of biofilm mass, similar to that delineated in the $C$. albicans biofilm. Similarly, in dual-species biofilms, mixed-culture biofilms treated with chelerythrine at $1 / 8$ MIC presented a flake-like structure, with a significant increase in porosity, while the biofilm biomass remarkably decreased in the 1/2 MIC group, as compared with the 
1/4 MIC group. These results showed chelerythrine at $1 / 2$ MIC could effectively inhibit the biofilms formation C. albicans and S. aureus mono- and dual-species.
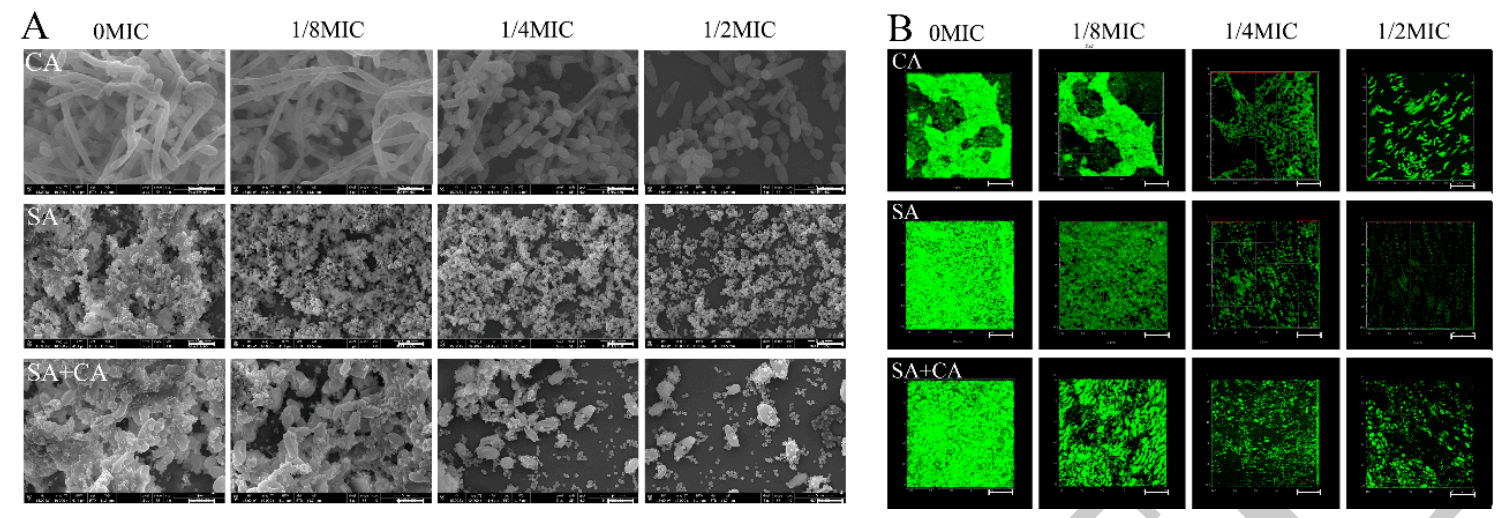

Figure 2. Inhibitory effects of sub-MIC chelerythrine on C. albicans (CA) and S. aureus (SA) mono- and dual-culture (SA+CA) biofilms, shown in field emission scanning electron microscopy (FESEM, (A)) and confocal laser scanning microscopy images (CLSM, (B)). Scale bars represent $5 \mu \mathrm{m}$ for FESEM and $10 \mu \mathrm{m}$ for CLSM, respectively.

3.4. eDNA Levels within S. aureus Mono-Species and C. albicans and S. aureus Dual-Species Biofilms Decreases in Response to Chelerythrine

To examine whether eDNA contributes to the observed decreased biofilm formation in response to chelerythrine treatment, CLSM image analyses were applied to investigate the in situ eDNA level, where eDNA was stained with a red fluorescent membrane impermeable DNA-binding stain PI, and SYTO 9, a green-fluorescent nucleic acid counterstain, was employed to stain cells of monoand dual-species. As displayed in Figure 3A, in S. aureus mono-species, as well as C. albicans and S. aureus dual-species biofilms, the red labeling is more intense in a chelerythrine-free biofilm than in a biofilm formed in the presence of chelerythrine, revealing chelerythrine exposure reduced the eDNA quantity in a dose dependent manner. In contrast, in a C. albicans mono-species biofilm, the red fluorescence was less visible in the presence or absence of chelerythrine, indicating that the matrix of C. albicans biofilms contains a small amount of eDNA. Moreover, in the case of S. aureus mono-species, as well as $C$. albicans and S. aureus dual-species biofilms, some scattered yellow colorations caused by the superposition of the green (bacterial cells) and red (eDNA) fluorescence were observed in both the control and 1/8 MIC-treated groups, suggesting that eDNA was mostly localized inside these biofilms. To further evaluate the relative changes of eDNA within biofilms formed by mono- and dual-species of C. albicans and S. aureus, the relative intensity of eDNA fluorescence was calculated. As displayed in Figure $3 \mathrm{~B}$, the relative fluorescence intensities of eDNA in untreated C. albicans and S. aureus monoand dual-species biofilms were significantly higher than those of biofilms of the 1/2 MIC-treated group $(100 \%, 100 \%$, and $100 \%$ versus $2.1 \% \pm 1.0 \%, 3.3 \% \pm 1.5 \%$, and $7.2 \% \pm 1.7 \%$, respectively; $p<0.001$ ). These results indicate that eDNA decrease contributes to a certain extent to chelerythrine-reduced biofilm formation by S. aureus mono-species and dual-species, while this situation was not suitable for C. albicans biofilms in response to chelerythrine. 

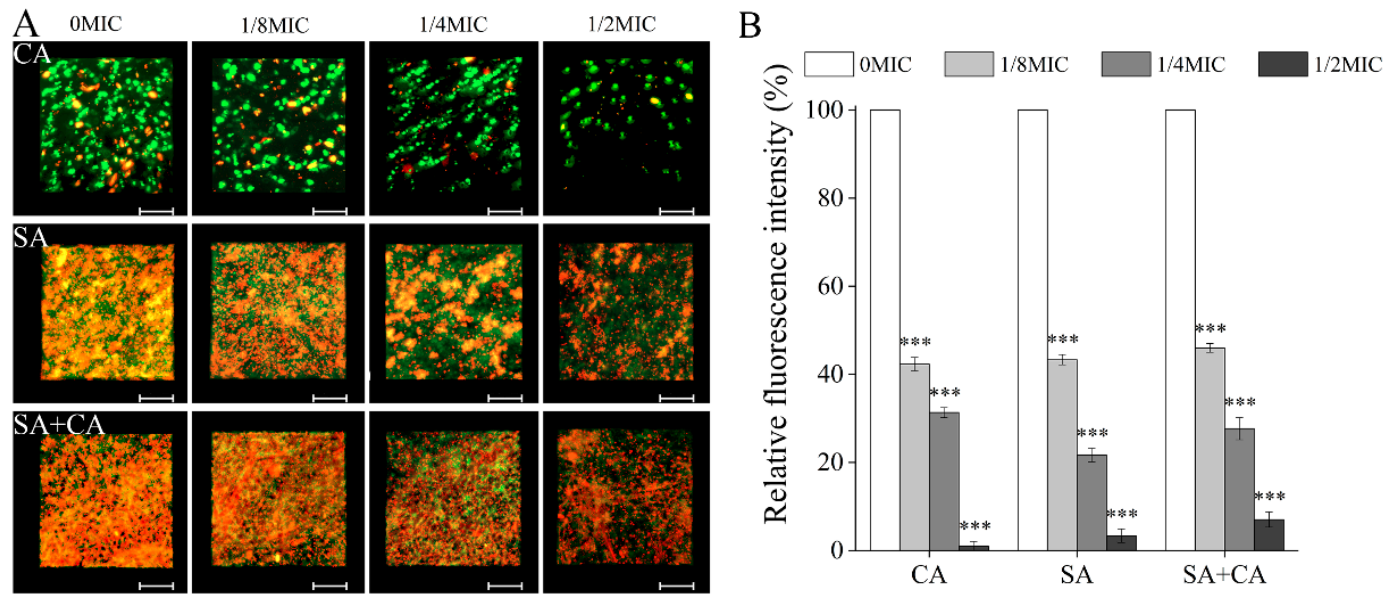

Figure 3. Effect of different concentrations of chelerythrine on the eDNA levels within C. albicans (CA) and S. aureus (SA) mono- and dual-species (SA+CA) biofilms by confocal laser scanning microscope. (A) eDNA was labelled with a red fluorescent membrane impermeable DNA-binding stain PI, and bacterial cells were stained with a green-fluorescent nucleic acid counterstain STYO 9. Scale bars represent $10 \mu \mathrm{m}$. (B) The relative fluorescence intensity of eDNA in biofilms of each treatment group was plotted against that in untreated group by measuring red fluorescence intensities using KS 400 version 3.0 software. Bars represent the standard deviation $(n=3) .{ }^{* *} p<0.001$.

3.5. Chelerythrine Reduced the Biofilm Formation of C. albicans and S. aureus Mono- and Dual-Species by Mediating Extracellular Proteins Levels

To visualize the inhibitory effect of chelerythrine on C. albicans and S. aureus mono- and dual-species biofilms, the biofilm matrices were stained with two fluorescent dyes (SYPRO Ruby and STYO 9) and assessed by confocal laser scanning microscopy (CLSM). According to Figure 4A, chelerythrine significantly reduced C. albicans and S. aureus mono- and dual-species biofilm formation with a dose-related change. Interestingly, in C. albicans mono-species biofilms, a large number of extracellular proteins were seen in the control group, whereas at 1/4 MIC-chelerythrine treated group the protein contents were remarkably reduced, suggesting that extracellular proteins could play a role in C. albicans biofilm formation. Similarly, in C. albicans and S. aureus dual-species biofilms exposed to 1/4 MIC-chelerythrine, the density of extracellular proteins was remarkably reduced, compared with the untreated control. In contrast, in S. aureus mono-species biofilms, extracellular protein content of $S$. aureus biofilms decreased with the rise of chelerythrine concentration, but extracellular proteins were evenly embedded in the biofilm in the mosaic-like pattern. Imaging measurements demonstrated that the relative fluorescence intensities of proteins of untreated C. albicans and S. aureus mono- and dual-species biofilms were statistically higher than those of biofilms of $1 / 2 \mathrm{MIC}$ treated group $(100 \%$, $100 \%$, and $100 \%$ versus $2.2 \% \pm 1.3 \%, 3.2 \% \pm 1.6 \%$ and $0.6 \% \pm 0.5 \%$, respectively; $p<0.001$ ) (Figure $4 \mathrm{~B}$ ). These results demonstrate that a decrease in the content of extracellular proteins contributed to a certain extent to chelerythrine-decreased biofilm formation by mono- and dual-species. 

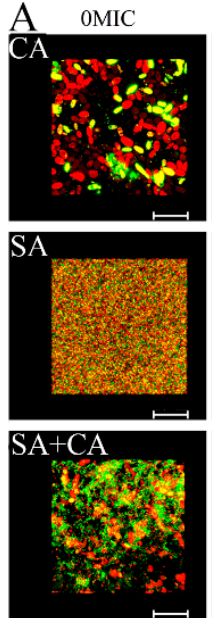
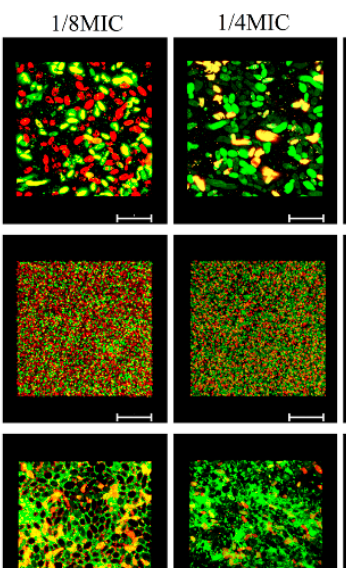

Wrates
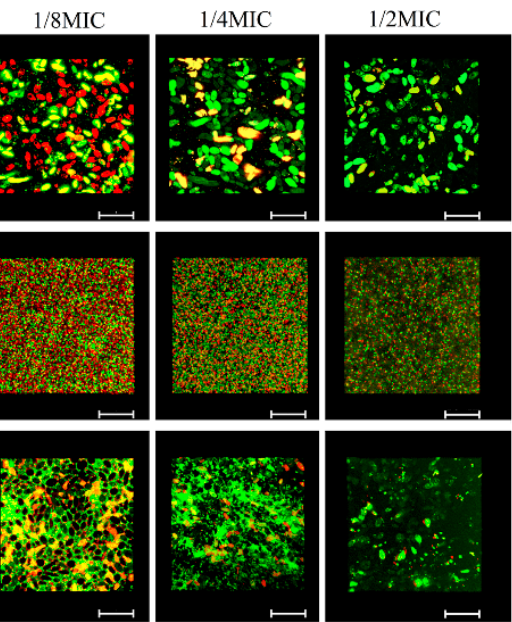

$\mathrm{B}$

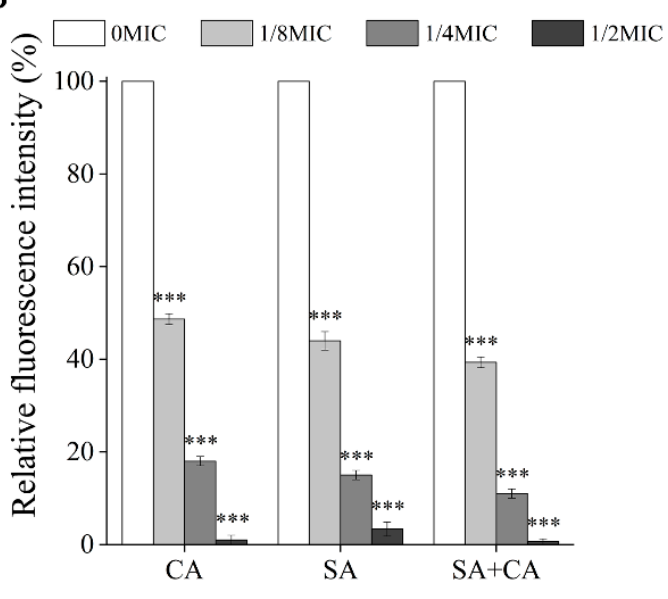

Figure 4. Effect of different concentrations of chelerythrine on C. albicans (CA) and S. aureus (SA) monoand dual-species (SA+CA) biofilm matrix structure. (A) Extracellular proteins were stained with a red fluorescent stain SYPRO Ruby, and C. albicans and S. aureus cells were stained with a green-fluorescent nucleic acid counterstain STYO 9. Scale bars represent $10 \mu \mathrm{m}$. (B) The relative fluorescence intensity of extracellular proteins in biofilms of each treatment group was plotted against that in untreated group by measuring red fluorescence intensities using KS 400 version 3.0 software. Bars represent the standard deviation $(n=3) .{ }^{* *} p<0.001$.

3.6. Chelerythrine Decreases the Biofilm Formation of C. albicans and S. aureus Mono- and Dual-Species by Mediating Extracellular Polysaccharides Levels

To assess the inhibitory effect of chelerythrine against $C$. albicans and S. aureus mono- dual-species biofilms, the biofilm matrix was stained with a combination of fluorescent stains wheat germ agglutinin and lipophilic membrane dye FM 4-64, and then evaluated by CLSM. As demonstrated in Figure 5A, in untreated C. albicans mono-species biofilms, CLSM images showed the green fluorescence occupied a significant part of the biovolume, suggesting a large number of extracellular polysaccharides in C. albicans biofilms. Interestingly, in the $1 / 8$ MIC chelerythrine-treated C. albicans and S. aureus mono-species biofilms, a significant reduction in the content of the extracellular polysaccharides was observed, with a dose-independent manner. In contrast, in dual-species biofilms of $C$. albicans and $S$. aureus, extracellular polysaccharides levels decreased in a dose-dependent manner, and a large number of yellow fluorescence was distributed throughout the whole biofilm structure in the untreated group, indicating that mixed-culture contributed to extracellular polysaccharides production. Moreover, Figure 5B shows that the relative fluorescence intensities of polysaccharides in untreated C. albicans and S. aureus mono- and dual- species biofilms were significantly higher than those in biofilms of the $1 / 2$ MIC-treated groups $(100 \%, 100 \%$, and $100 \%$ versus $0.8 \% \pm 0.6 \%, 4.5 \% \pm 1.6 \%$, and $8.6 \% \pm 1.6 \%$, respectively; $p<0.001)$. 
A $0 \mathrm{MIC}$
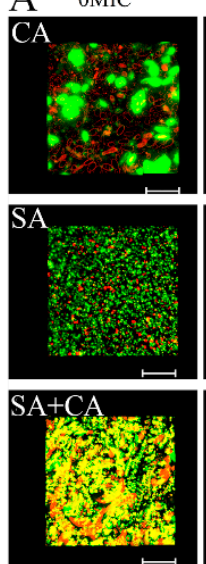
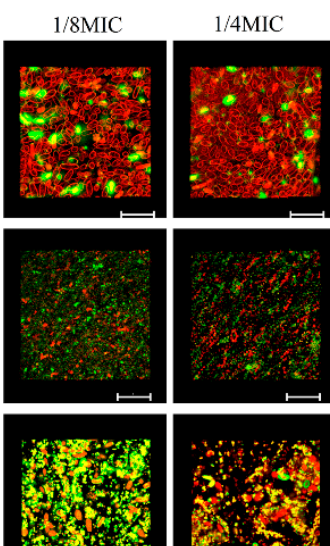

2.
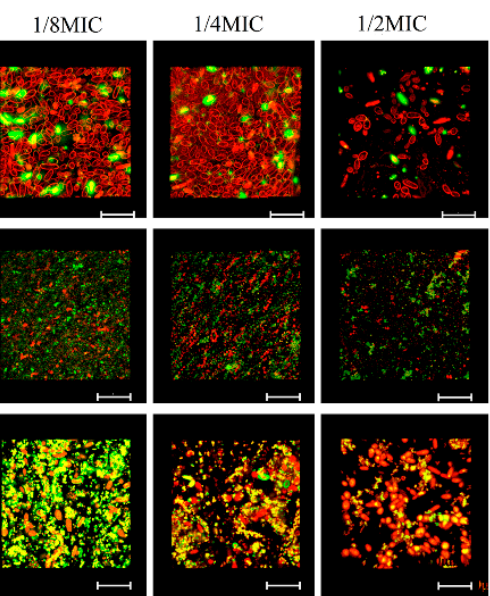

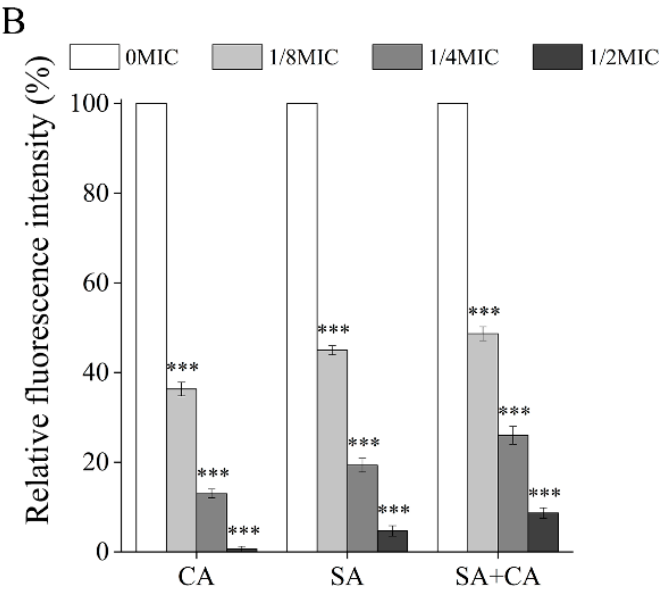

$/ 2 \mathrm{MIC}$

Figure 5. Effects of different concentrations of chelerythrine on the levels of extracellular polysaccharides inside C. albicans (CA) and S. aureus (SA) mono- and dual-species (SA+CA) biofilms. (A) Extracellular polysaccharides were stained with a green fluorescent stain wheat germ agglutinin, and C. albicans and S. aureus cells were labelled with a red lipophilic membrane dye FM 4-64. Scale bars represent $10 \mu \mathrm{m}$. (B) The relative fluorescence intensity of extracellular polysaccharides in biofilms of each treatment group was plotted against that in untreated group by measuring green fluorescence ratios using KS 400 version 3.0 software. Bars represent the standard deviation $(n=3)$. ${ }^{* * *} p<0.001$.

\subsection{Chelerythrine Treatment Reduces Mono- and Dual-Species Biofilms Tolerance to Gatifloxacin}

Next, to assess the biofilms exposed to chelerythrine tolerance to antibiotics, gatifloxacin diffusion within mono- and dual-species biofilms was comparatively monitored using CLSM. As demonstrated in Figure 6, in untreated mono- and dual-species biofilms, we observed that gatifloxacin was confined to the outer periphery of the biofilm with minimal to no penetration into the biofilm. Also, under treatment with chelerythrine at 1/8 MIC conditions, a limited penetration of gatifloxacin into the mono- and dual-species biofilms was observed. In contrast, an increased penetration of gatifloxacin into the monoand dual-species biofilms was found in the $1 / 4$ MIC treatment group. Notably, under the concentration of chelerythrine at $1 / 2 \mathrm{MIC}$, it is obviously seen that gatifloxacin penetration was full within the monoand dual-species biofilms, reaching the basal layers.
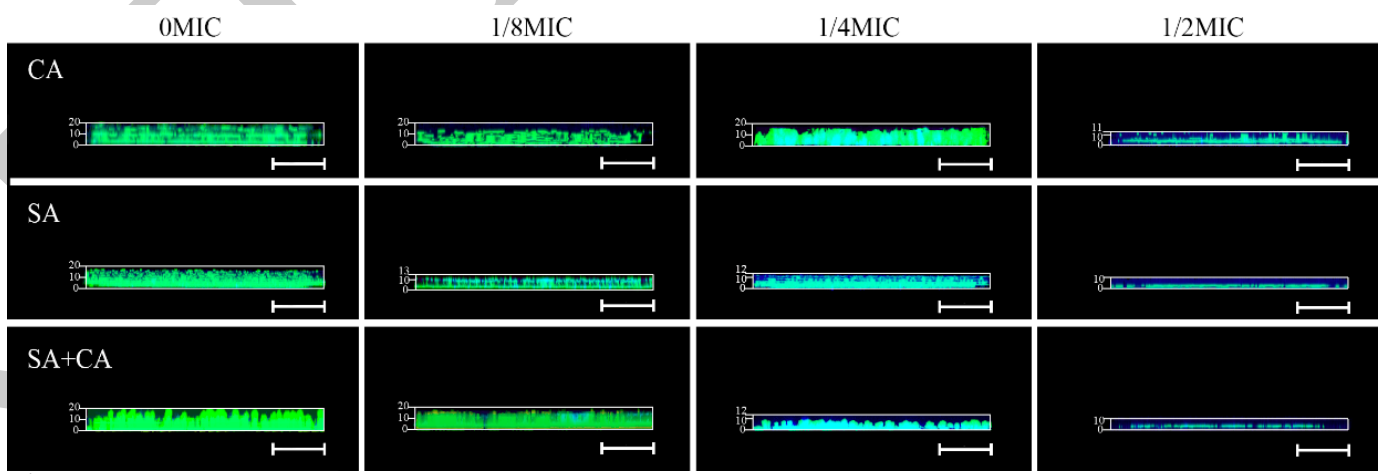

Figure 6. Representative CLSM images assessing diffusion of gatifloxacin within C. albicans (CA) and S. aureus (SA) mono- and dual-species (SA+CA) biofilms formed in the presence of chelerythrine. After mono- and dual-species biofilms were grown for $24 \mathrm{~h}$ supplemented without or with chelerythrine of different concentrations, $0.4 \mathrm{mg} / \mathrm{mL}$ gatifloxacin was added into the medium. Following $5 \mathrm{~h}$ gatifloxacin diffusion, the biofilms were visualized using CLSM. Biofilms were stained with SYTO 9 for biofilms (green) and the intrinsic fluorescence of gatifloxacin (blue). Scale bars represent $10 \mu \mathrm{m}$. 
3.8. Chelerythrine Eradicates Efficiently Preformed Biofilms of C. albicans and S. aureus Mono- and Dual-Species

To confirm the biofilm eradication activity of chelerythrine, we evaluated the amount of and morphological changes to biofilms formed by mono- and dual-species influenced by exposure to chelerythrine at four sub-MIC concentrations, using the crystal violet staining assay and FESEM. As displayed in Figure 7A, the biofilm biomass of $C$. albicans and S. aureus mono-species was significantly reduced by treatment with chelerythrine at 16 MIC $(p<0.001)$, while a similar result was achieved at 32 MIC in dual-species group $(p<0.001)$. In addition, the removal potential of chelerythrine for monoand dual-species mature biofilms was determined by FESEM. According to Figure 8, the eradication effect of biofilms enhanced as the chelerythrine concentration increased. Specifically, mature biofilms of C. albicans without chelerythrine treatment gave rise to dense aggregates, whereas $>90 \%$ biofilms were eradicated when exposed to 16 or 32 MIC treatment. Similarly, treatment of S. aureus mono-species and dual-species biofilms with 32 MIC of chelerythrine resulted in almost complete degradation of biofilm architecture, and disaggregated the C. albicans and S. aureus cells in dual-species biofilms. The results were consistent with those obtained using the CVSA. Moreover, the results from CFU recovery displayed a remarkable decrease in C. albicans and S. aureus survival in a concentration-dependent manner when mono and mixed biofilms were treated with chelerythrine. Notably, $32 \mathrm{MIC}$ chelerythrine treatment significantly diminished the ability of C. albicans and S. aureus mono- and dual-species biofilms to confer protection against viability of microbial cells (Figure 7B).

A

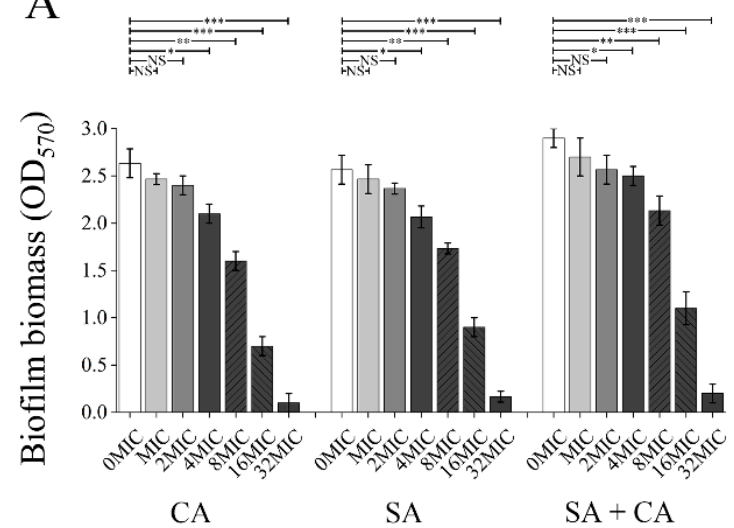

Chelerythrine concentration

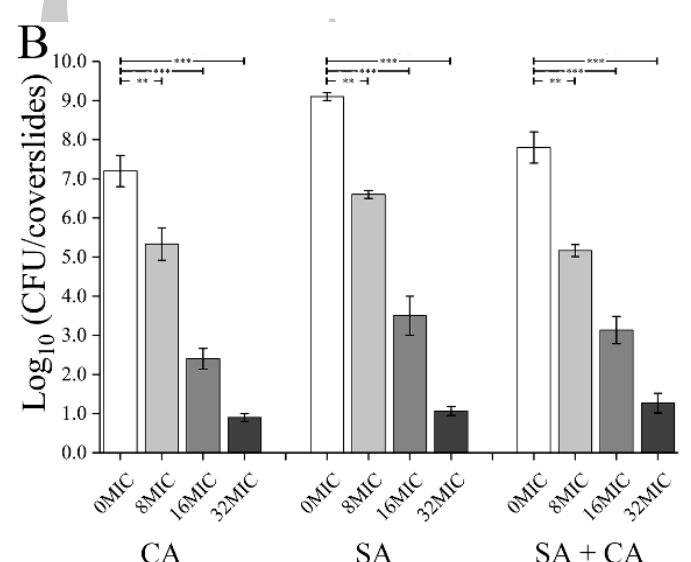

Chelerythrine concentration

Figure 7. Assessment of eradication effects of different concentrations of chelerythrine on C. albicans (CA) and S. aureus (SA) mono- and dual-species (SA+CA) mature biofilm biomass. (A) The biofilm biomass was assessed using crystal violet staining assay. (B) Mature mono and mixed species biofilms were treated with different concentrations of chelerythrine, and fungal or bacterial cell viability was examined by CFU recovery. Values represent the means of triplicate measurements. Bars represent the standard deviation $(n=3) .{ }^{*} p<0.05 ;{ }^{* *} p<0.01 ;{ }^{* *} p<0.001$; NS, not significant. 

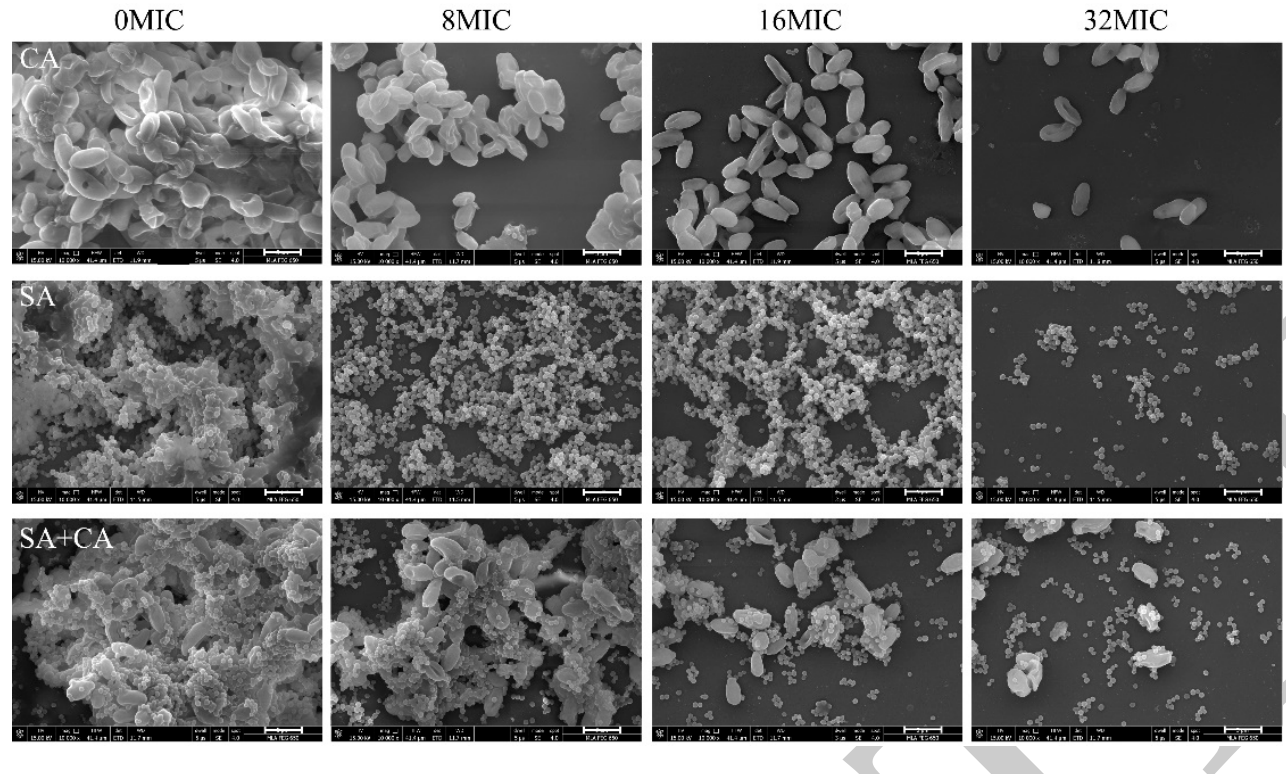

Figure 8. Scanning electron microscopy images of $C$. albicans (CA) and S. aureus (SA) monoand dual-species $(\mathrm{SA}+\mathrm{CA})$ mature biofilms after treatment with four MIC-fold concentrations of chelerythrine. Each field of vision was magnified 10,000x. Scale bars represent $5 \mu \mathrm{m}$.

\subsection{Chelerythrine Inhibits the Hyphae Formation of C. albicans}

The effect of chelerythrine on C. albicans hypha formation was further evaluated by incubating C. albicans in an RPMI-1640 medium supplemented with chelerythrine. As shown in Figure 9, massive $C$. albicans hyphae were observed in the untreated group, and C. albicans formed strong peripheral filaments. However, the growth of hyphae was markedly attenuated with the addition of chelerythrine at concentrations of 1/4 MIC and 1/2 MIC. Notably, hyphal formation was almost completely blocked by chelerythrine at the concentration of an MIC, where C. albicans cells were maintained as yeasts. These results reveal that chelerythrine inhibited the hypha formation in a concentration-dependent manner.
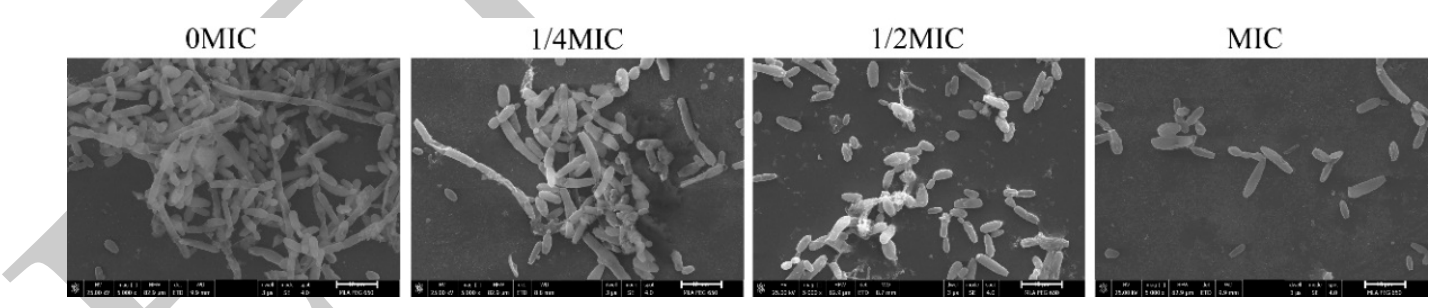

Figure 9. The effect of chelerythrine on the hyphal growth of C. albicans. Candida albicans cells were grown in an RPMI-1640 medium at the indicated concentration of chelerythrine at $37^{\circ} \mathrm{C}$ for $24 \mathrm{~h}$. Each field of vision was magnified 5000×. Scale bars represent $10 \mu \mathrm{m}$.

\subsection{Chelerythrine Induce the Hypha-to-Yeast Transition in C. albicans}

The effect of chelerythrine on the hypha-to-yeast transition was examined by FESEM. As shown in Figure 10, some untreated cells were found to have massive hyphae. In contrast, 1/2 MIC chelerythrine resulted in the formation of shorter hyphae, and a significant population of yeast forms was observed after treatment with MIC of chelerythrine for $5 \mathrm{~h}$. Notably, at 2 MIC, chelerythrine treatment concentration cells were completely devoid of hyphae and remains in yeast form. 
OMIC

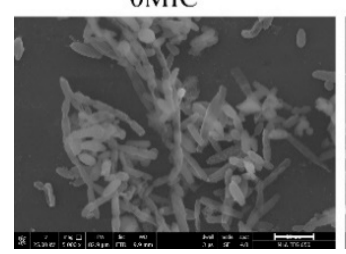

$1 / 2 \mathrm{MIC}$

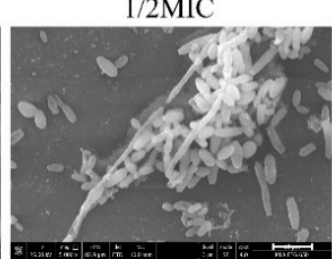

MIC

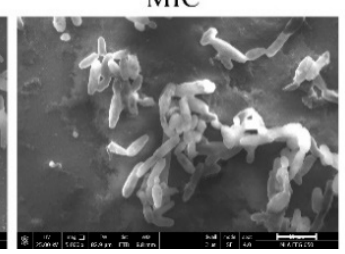

$2 \mathrm{MIC}$

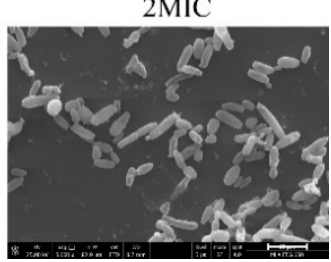

Figure 10. The effect of chelerythrine on C. albicans mature hyphae. Candida albicans mature hypha were formed in RPMI-1640 for $24 \mathrm{~h}$ and treated with indicated concentrations of chelerythrine for $5 \mathrm{~h}$ at $37^{\circ} \mathrm{C}$. Each field of vision was magnified 5000×. Scale bars represent $10 \mu \mathrm{m}$.

\section{Discussion}

The mixed infection of $C$. albicans and S. aureus occurred frequently, which can lead to several diseases with a high mortality, rather than a single-species infection [26]. Synergistic interaction between C. albicans and S. aureus contributed to the development of polymicrobial biofilm communities, resulting in strong resistance to antibiotics, highlighting the need to find new antimicrobial agents [27]. In this study, we firstly examined the chelerythrine-mediated growth inhibition against mono- and dual-species planktic cells of $C$. albicans and S. aureus. The results showed MICs of chelerythrine were $4 \mu \mathrm{g} / \mathrm{mL}$ for both single $C$. albicans and S. aureus culture, while the MIC for a dual culture of $C$. albicans and $S$. aureus was $6 \mu \mathrm{g} / \mathrm{mL}$. By contrast, He et al. showed that chelerythrine isolated from root of Toddalia asiatica (Linn) Lam exhibited a moderate growth-inhibitory effect against planktonic cells of S. aureus 25923 , methicillin-resistant $S$. aureus, and extended spectrum $\beta$-lactamase $S$. aureus, and the MICs of chelerythrine against these three bacteria were all $156 \mu \mathrm{g} / \mathrm{mL}$ [13]. The data were higher than our results, which may be due to the purity of extracted chelerythrine extracted from Toddalia asiatica (Linn) Lam.

Moreover, biofilms protect embedded microbial cells against an in-principle adequate antimicrobials therapy, frequently resulting in treatment failure and relapsing infections. To further evaluate antibiofilm activities of chelerythrine against mono- and dual-species, a combination of CVSA, CFU enumeration, FESEM, and CLSM was employed. These results indicated that the MBIC $_{905}$ of chelerythrine for C. albicans and S. aureus mono- and dual-species were 2 and $3 \mu \mathrm{g} / \mathrm{mL}$, respectively. Notably, the inhibitory concentration of chelerythrine against biofilm formation of monoand dual-culture models of C. albicans and S. aureus was less than that of previous reports, where Shin and Eom reported that zerumbone remarkably inhibited mono- and dual-species biofilms formed by C. albicans and S. aureus at $64 \mu \mathrm{g} / \mathrm{mL}$ [28]. Similarly, She et al. showed that auranofin could effectively inhibit S. aureus and C. albicans mono- and dual-biofilm formation in vitro at $8 \mu \mathrm{g} / \mathrm{mL}$ [29]. Collectively, it can be concluded that chelerythrine had potent antibiofilm activities against a monoand dual-culture of C. albicans and S. aureus. Interestingly, in contrast to the growth inhibitory potency against planktonic cells, the biofilm formation of C. albicans and S. aureus mono- and dual-species was more susceptible to chelerythrine with subminimal inhibitory concentrations (sub-MICs), respectively; however, biofilms formed over $24 \mathrm{~h}$ on a glass coverslips surface were more resistant to chelerythrine than were planktonic cells. The results are in agreement with previous studies, wherein sub-MICs of antimicrobial agents can suppress biofilm formation by affecting initial microbial adherence to surfaces [30,31]. It is important to highlight that chelerythrine displayed a significant inhibitory effect at the sub-MICs for biofilm growth, thus demonstrating its potential to minimize microbial resistance when combined with low-dose antimicrobials.

Next, we further revealed the profile of extracellular proteins, polysaccharides, and eDNA of biofilms formed by mono- and dual-cultures of C. albicans and S. aureus after treatment with chelerythrine at sub-MIC. To this end, CLSM, in conjugation with five different fluorescent dyes, was applied to differentiate bacterial cells from proteins, polysaccharides, and eDNA within the biofilm matrix. The results confirmed that chelerythrine reduced biofilm formation by mediating mainly polysaccharides and eDNA levels for an S. aureus single culture, while for a $C$. albicans single 
culture, chelerythrine inhibited biofilm formation by decreasing mainly proteins and polysaccharides levels. These data were in line with previous reports that important components of S. aureus biofilm are extracellular polysaccharides and eDNA, while the $C$. albicans biofilm matrix is predominantly composed of proteins (55\%), carbohydrates (25\%), lipids (15\%), and eDNA $(5 \%)$ [32,33]. In agreement with these findings, for dual cultured of $C$. albicans and S. aureus, chelerythrine diminished biofilm formation by decreasing simultaneously extracellular proteins, polysaccharides, and eDNA levels in a dose-dependent manner. Interestingly, for chelerythrine-treated C. albicans mono-species and dual-species biofilms, the amount of eDNA was correlated positively with biofilm-forming capacity, suggesting that an abundant amount of eDNA was essential for the development of robust biofilms. In contrast, the importance of eDNA in biofilms may be overlooked in chelerythrine-reduced biofilm formation by $S$. aureus mono-species.

Meanwhile, hindered penetration of antibiotics through biofilms is one of the contributing factors to the remarkable increase in bacterial tolerance to antibiotics [34]. Here, we investigated the tolerance of biofilms of mono- and dual-culture of $C$. albicans and S. aureus after treatment with chelerythrine to antibiotics diffusion. To this end, gatifloxacin with intrinsic fluorescence in the range deep ultra-violet wavelength was employed to visualize the antibiotic diffusion through biofilm by CLSM. The results showed that gatifloxacin had minimal penetration into the matrix in the presence of chelerythrine at low concentration of $1 / 8 \mathrm{MIC}$, while gatifloxacin diffusion in both mono- and dual-species biofilms were increased significantly when exposed to chelerythrine at high concentrations of $1 / 2 \mathrm{MIC}$, revealing that the integrity of the mono- and dual-species biofilms was disrupted by chelerythrine, thereby enhancing the osmotic of biofilms.

After verifying the antimicrobial and antibiofilm activities of chelerythrine, we investigated mature biofilm eradication activities of chelerythrine against mono- dual-microbial biofilms. It has been acknowledged that once the biofilm is well formed, the resistance to antimicrobials can be extremely increased. In the present study, chelerythrine could efficiently eradicate $96.4 \%$ and $92.3 \% 24 \mathrm{~h}$ mature biofilm formed by mono-cultures of C. albicans and S. aureus at concentrations of $128 \mu \mathrm{g} / \mathrm{mL}$, and $90.7 \%$ 24-h mature biofilm of dual-culture at concentrations of $192 \mu \mathrm{g} / \mathrm{mL}$, respectively. In contrast, Shin and Eom reported that treatment with $500 \mu \mathrm{g} / \mathrm{mL}$ of zerumbone resulted in a dramatic elimination of preformed C. albicans biofilm by $96.3 \%$ [28]. On the other hand, in the case of preformed S. aureus, dual-species biofilms were reduced by $49 \%$ and $39.4 \%$ at concentrations of $500 \mu \mathrm{g} / \mathrm{mL}$, suggesting that chelerythrine was more effective in eradicating mono- and dual-species of $C$. albicans and S. aureus preformed biofilm than zerumbone.

Since hyphal growth plays an important role in C. albicans infection by mediating dissemination of $C$. albicans to the host tissues, the inhibition of $C$. albicans to hyphal growth forms is a significant finding [35]. Our results present that hyphal growth was remarkably inhibited at $4 \mu \mathrm{g} / \mathrm{mL}$ concentration of chelerythrine, showing a robust activity of chelerythrine against hyphal growth. In addition, the effect of chelerythrine on the mature hyphae of $C$. albicans was also tested. Hyphae of $C$. albicans in the presence of $4 \mu \mathrm{g} / \mathrm{mL}$ were shortened and majorly shifted to yeast state at $8 \mu \mathrm{g} / \mathrm{mL}$ concentration of chelerythrine used. A reason for shortening of $C$. albicans hyphae may be due to the reversible morphological plasticity between yeast and hyphal forms in response to chelerythrine. Similar results for reverse morphogenesis were observed with gymnemic acid, which transforms the hyphal cells of C. albicans into yeast cells [36].

In addition, previous reports demonstrated that the anti-cancer effects of chelerythrine have been studied both in vitro and in vivo [37], thus attracting wide interest for its potential clinical applications. For example, NK109, a chelerythrine analogue, is in Phase 2 trial in Japan and 7-hydroxystaurosporine, a PKC inhibitor, in Phase 1 trials in the United States [38]. Further, Kosina et al. evaluated the effects of daily administration of the extract from Macleya cordata in the diet on the health status of swine. After 90 day administration the highest chelerythrine and sanguinarine (1:3) retention was detected in the gingiva $(0.55 \mu \mathrm{g} / \mathrm{g})$ and liver $(0.15 \mu \mathrm{g} / \mathrm{g})$, and treated animals did not demonstrate any results of hematological, biochemical, or histological assay different from controls [39]. Collectively, all of these 
results suggest that chelerythrine shows promise as a potential antimicrobial and antibiofilm agent for clinical effective treatments of mono- and mixed-species and/or biofilm-associated infections both in vitro and in vivo.

Author Contributions: Conceptualization and methodology, W.Q.; software and validation, J.Z.; writing-original draft preparation, W.W.; formal analysis, M.L.; investigation, Y.F.; resources, X.L.; data curation, T.W.; visualization and validation, Y.L. All authors have read and agreed to the published version of the manuscript.

Funding: This work was supported by the National Natural Science Foundation of China $(11975177,11575149)$, the Science and Technology Program of China Selenium Industry Research Institute (2018FXZX03-15), and the Xi'an Weiyang District Science and technology project [201926].

Conflicts of Interest: The authors declare no conflict of interest.

\section{References}

1. Diekema, D.J.; Messer, S.A.; Brueggemann, A.B.; Coffman, S.L.; Doern, G.V;; Herwaldt, L.A.; Pfaller, M.A. Epidemiology of candidemia: 3-year results from the emerging infections and the epidemiology of lowa organisms study. J. Clin. Microbiol. 2002, 40, 1298-1302. [CrossRef] [PubMed]

2. Magill, S.S.; O’Leary, E.; Janelle, S.J.; Thompson, D.L.; Dumyati, G.; Nadle, J.; Wilson, L.E.; Kainer, M.A.; Lynfield, R.; Greissman, S.; et al. Changes in prevalence of health care-associated infections in U.S. hospitals. N. Engl. J. Med. 2018, 379, 1732-1744. [CrossRef] [PubMed]

3. Dennis, E.K.; Garneau-Tsodikova, S. Synergistic combinations of azoles and antihistamines against Candida species in vitro. Med. Mycol. 2019, 57, 874-884. [CrossRef] [PubMed]

4. Peters, B.M.; Jabra-Rizk, M.A.; O’May, G.A.; Costerton, J.W.; Shirtliff, M.E. Polymicrobial interactions: Impact on pathogenesis and human disease. Clin. Microbiol. Rev. 2012, 25, 193-213. [CrossRef]

5. Pan, J.; Zhao, J.; Jiang, N. Oral cavity infection: An adverse effect after the treatment of oral cancer in aged individuals. J. Appl. Oral. Sci. 2014, 22, 261-267. [CrossRef]

6. Donlan, R.M.; Costerton, J.W. Biofilms: Survival mechanisms of clinically relevant microorganisms. Clin. Microbiol. Rev. 2002, 15, 167-193. [CrossRef]

7. Carolus, H.; Van Dyck, K.; Van Dijck, P. Candida albicans and Staphylococcus species: A threatening twosome. Front. Microbiol. 2019, 10, 2162. [CrossRef]

8. Kong, E.F.; Tsui, C.; Kucharikova, S.; Andes, D.; Van Dijck, P.; Jabra-Rizk, M.A. Commensal protection of Staphylococcus aureus against antimicrobials by Candida albicans biofilm matrix. MBio 2016, 7, e01365-16. [CrossRef]

9. Koo, H.; Andes, D.R.; Krysan, D.J. Candida-streptococcal interactions in biofilm-associated oral diseases. PLoS Pathog. 2018, 14, e1007342. [CrossRef]

10. Bowen, W.H.; Burne, R.A.; Wu, H.; Koo, H. Oral biofilms: Pathogens, matrix, and polymicrobial interactions in microenvironments. Trends Microbiol. 2018, 26, 229-242. [CrossRef]

11. Rodrigues, M.E.; Gomes, F.; Rodrigues, C.F. Candida spp./bacteria mixed biofilms. J. Fungi $2019,6,5$. [CrossRef] [PubMed]

12. Desai, J.V.; Mitchell, A.P.; Andes, D.R. Fungal biofilms, drug resistance, and recurrent infection. Cold Spring Harb. Perspect. Med. 2014, 4, a019729. [CrossRef] [PubMed]

13. Niu, X.F.; Zhou, P.; Li, W.F.; Xu, H.B. Effects of chelerythrine, a specific inhibitor of cyclooxygenase-2, on acute inflammation in mice. Fitoterapia 2011, 82, 620-625. [CrossRef]

14. Miao, F.; Yang, X.J.; Ma, Y.N.; Zheng, F.; Song, X.P.; Zhou, L. Structural modification of sanguinarine and chelerythrine and their in vitro acaricidal activity against Psoroptes cuniculi. Chem. Pharm. Bull. 2012, 60, 1508-1513. [CrossRef]

15. He, N.; Wang, P.Q.; Wang, P.Y.; Ma, C.Y.; Kang, W.Y. Antibacterial mechanism of chelerythrine isolated from root of Toddalia asiatica (Linn) Lam. BMC Complement. Altern. Med. 2018, 18, 261. [CrossRef] [PubMed]

16. Kang, Y.J.; Yi, Y.L.; Zhang, C.; Wu, S.Q.; Shi, C.B.; Wang, G.X. Bioassay-guided isolation and identification of active compounds from Macleaya microcarpa (Maxim) Fedde against fish pathogenic bacteria. Aquac. Res. 2013, 44, 1221-1228. [CrossRef] 
17. Tantapakul, C.; Phakhodee, W.; Ritthiwigrom, T.; Yossathera, K.; Deachathai, S.; Laphookhieo, S. Antibacterial compounds from Zanthoxylum rhetsa. Arch. Pharmacal Res. 2012, 35, 1139-1142. [CrossRef]

18. Gong, Y.; Li, S.; Wang, W.; Li, Y.; Ma, W.; Sun, S. In vitro and in vivo activity of chelerythrine against Candida albicans and underlying mechanisms. Future Microbiol. 2019, 14, 1545-1557. [CrossRef]

19. Clinical and Laboratory Standards Institute (CLSI). Reference Method for Broth Dilution Antifungal Susceptibility Testing of Yeasts; CLSI: Wayne, PA, USA, 2008.

20. Clinical and Laboratory Standards Institute (CLSI). Methods for Dilution Antimicrobial Susceptibility Tests for Bacteria That Grow Aerobically; CLSI: Wayne, PA, USA, 2012.

21. Li, X.; Yin, L.; Ramage, G.; Li, B.; Tao, Y.; Zhi, Q.; Lin, H.; Zhou, Y. Assessing the impact of curcumin on dual-species biofilms formed by Streptococcus mutans and Candida albicans. Microbiologyopen 2019, 8, e937. [CrossRef]

22. Rho, D.; Chauret, N.; Laberge, N.; Archambault, J. Growth characteristics of Sanguinaria canadensis L. cell suspensions and immobilized cultures for production of benzophenanthridine alkaloids. Appl. Microbiol. Biotechnol. 1992, 36, 611-617. [CrossRef]

23. Wright, C.S. Structural comparison of the two distinct sugar binding sites in wheat germ agglutinin isolectin II. J. Mol. Biol. 1984, 178, 91-104. [CrossRef]

24. Jegal, U.; Lee, J.H.; Lee, J.; Jeong, H.; Kim, M.J.; Kim, K.H. Ultrasound-assisted gatifloxacin delivery in mouse cornea, in vivo. Sci. Rep. 2019, 9, 15532. [CrossRef] [PubMed]

25. Qian, W.; Zhang, J.; Wang, W.; Wang, T.; Liu, M.; Yang, M.; Sun, Z.; Li, X.; Li, Y. Antimicrobial and antibiofilm activities of paeoniflorin against carbapenem-resistant Klebsiella pneumoniae. J. Appl. Microbiol. 2019, 128, 401-413. [CrossRef] [PubMed]

26. Ikeh, M.A.C.; Fidel, P.L., Jr.; Noverr, M.C. Identification of specific components of the eicosanoid biosynthetic and signaling pathway involved in pathological inflammation during intra-abdominal infection with Candida albicans and Staphylococcus aureus. Infect. Immun. 2018, 86, e00144-18. [CrossRef]

27. Li, H.; Zhang, C.Q.; Liu, P.; Liu, W.G.; Gao, Y.; Sun, S.J. In vitro interactions between fluconazole and minocycline against mixed cultures of Candida albicans and Staphylococcus aureus. J. Microbiol. Immunol. 2015, 48, 655-661. [CrossRef]

28. Shin, D.S.; Eom, Y.B. Efficacy of zerumbone against dual-species biofilms of Candida albicans and Staphylococcus aureus. Microb. Pathog. 2019, 137, 103768. [CrossRef]

29. She, P.F.; Liu, Y.Q.; Wang, Y.X.; Tan, F.; Luo, Z.; Wu, Y. Antibiofilm efficacy of the gold compound auranofin on dual species biofilms of Staphylococcus aureus and Candida sp. J. Appl. Microbiol. 2019, 128, 88-101. [CrossRef]

30. Yang, X.; Sha, K.; Xu, G.; Tian, H.; Wang, X.; Chen, S.; Wang, Y.; Li, J.; Chen, J.; Huang, N. Subinhibitory concentrations of allicin decrease uropathogenic Escherichia coli (UPEC) biofilm formation, adhesion ability, and swimming motility. Int. J. Mol. Sci. 2016, 17, 979. [CrossRef]

31. Qian, W.; Liu, M.; Fu, Y.; Zhang, J.; Liu, W.; Li, J.; Li, X.; Li, Y.; Wang, T. Antimicrobial mechanism of luteolin against Staphylococcus aureus and Listeria monocytogenes and its antibiofilm properties. Microb. Pathog. 2020, 142, 104056. [CrossRef]

32. Lopez-Ribot, J.L. Large-scale biochemical profiling of the Candida albicans biofilm matrix: New compositional, structural, and functional insights. Mbio 2014, 5, e01781-14. [CrossRef]

33. Sugimoto, S.; Sato, F.; Miyakawa, R.; Chiba, A.; Onodera, S.; Hori, S.; Mizunoe, Y. Broad impact of extracellular DNA on biofilm formation by clinically isolated methicillin-resistant and -sensitive strains of Staphylococcus aureus. Sci. Rep. 2018, 8, 2254. [CrossRef] [PubMed]

34. Teirlinck, E.; Xiong, R.H.; Brans, T.; Forier, K.; Fraire, J.; Van Acker, H.; Matthijs, N.; De Rycke, R.; De Smedt, S.C.; Coenye, T.; et al. Laser-induced vapour nanobubbles improve drug diffusion and efficiency in bacterial biofilms. Nat. Commun. 2018, 9, 4518. [CrossRef] [PubMed]

35. Haque, F.; Alfatah, M.; Ganesan, K.; Bhattacharyya, M.S. Inhibitory effect of sophorolipid on Candida albicans biofilm formation and hyphal growth. Sci. Rep. 2016, 6, 23575. [CrossRef] [PubMed]

36. Vediyappan, G.; Dumontet, V.; Pelissier, F.; d'Enfert, C. Gymnemic acids inhibit hyphal growth and virulence in Candida albicans. PLoS ONE 2013, 8, e74189. [CrossRef] [PubMed]

37. Kumar, S.; Tomar, M.S.; Acharya, A. Chelerythrine delayed tumor growth and increased survival duration of Dalton's lymphoma bearing BALB/c H(2d) mice by activation of NK cells in vivo. J. Cancer Res. Ther. 2015, 11, 904-910. [CrossRef] 
38. Chmura, S.J.; Dolan, M.E.; Cha, A.; Mauceri, H.J.; Kufe, D.W.; Weichselbaum, R.R. In vitro and in vivo activity of protein kinase $C$ inhibitor chelerythrine chloride induces tumor cell toxicity and growth delay in vivo. Clin. Cancer Res. 2000, 6, 737-742.

39. Kosina, P.; Walterova, D.; Ulrichova, J.; Lichnovsky, V.; Stiborova, M.; Rydlova, H.; Vicar, J.; Krecman, V.; Brabec, M.J.; Simanek, V. Sanguinarine and chelerythrine: Assessment of safety on pigs in ninety days feeding experiment. Food Chem. Toxicol. 2004, 42, 85-91. [CrossRef]

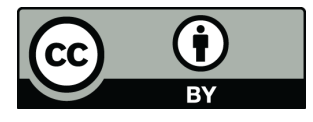

(C) 2020 by the authors. Licensee MDPI, Basel, Switzerland. This article is an open access article distributed under the terms and conditions of the Creative Commons Attribution (CC BY) license (http://creativecommons.org/licenses/by/4.0/). 